${ }^{1}$ Centro de Bioética, Facultad de Medicina, Clínica Alemana Universidad del Desarrollo. Santiago, Chile.

${ }^{2}$ Departamento de Bioética y Humanidades Médicas, Facultad de Medicina, Universidad de Chile. Santiago, Chile.

Los autores declaran no haber recibido ayuda financiera para la realización de este trabajo, más allá del sueldo correspondiente a su cargo académico institucional. Los autores declaran no tener conflictos de interés.

Recibido el 2 de octubre de 2017, aceptado el 15 de mayo de 2018.

Correspondencia a: Bernardo Aguilera baguilera@udd.cl

\section{Análisis ético de la entrega parcial de información al paciente para prevenir efectos nocebo}

\author{
BERNARDO AGUILERA ${ }^{1,2}$, JUAN PABLO BECA ${ }^{1}$
}

\section{Partial disclosure of information in the presence of presumable nocebo effects: an ethical analysis}

When prescribing a treatment, the physician should give truthful information about the likely benefits and the potential adverse effects, allowing the patient to make an autonomous decision about whether to take the treatment. However, the mere expectation of adverse effects may precipitate the corresponding symptoms. This is called "nocebo effect", which in contrast to the placebo effect, can lead to harm to the patient due to psychological factors. Nocebo effects are common and clinically significant, although often unnoticed. This situation generates conflicts in medical ethics guiding principles, namely the moral obligation to disclose all possible effects of the prescribed drug as opposed to the duty of avoiding the harm of side effects that are likely to occur in a case. In other words, the physician faces a dilemma between the due respect for autonomy and the duty of non-maleficence. This article reflects about this conflict, by exploring the limits of the principle of autonomy and how to balance it with the principle of non-maleficence. We suggest an interpretation of the principle of autonomy from a patient-centered perspective, suggesting that it is ethically sound to give a prudential, partial disclosure of information to the patient, for the sake of avoiding potential nocebo effects. The article concludes with some cautionary considerations to be considered about this decision.

(Rev Med Chile 2018; 146: 518-522)

Key words: Bioethics; Disclosure; Ethics, Medical; Informed Consent; Nocebo Effect; Truth Disclosure.

\section{Efecto nocebo y sus problemas éticos}

1 1 término "nocebo" fue acuñado en 1961 por Kennedy para denotar el equivalente negativo de los síntomas favorables que los pacientes suelen presentar tras ingerir un placebo $^{1}$. El efecto nocebo corresponde a síntomas y/o cambios fisiológicos de carácter indeseado o perjudicial, que se siguen de la administración de una sustancia (ya sea activa o inerte), pero que no son producto de la actividad biológica y farmacológica específica de dicha sustancia. Tampoco son, por lo tanto, catalogables como efectos adversos ${ }^{2,3}$. Para $\mathrm{Hahn}^{4}$, el factor común en estos casos es que la expectativa de presentar síntomas de enfermedad acaba causando estos síntomas. Por ejemplo, en un estudio del uso de finasteride para el tratamiento de adultos con hiperplasia prostática benigna, los pacientes que fueron previamente informados de posibles efectos adversos sexuales del fármaco reportaron haber experimentado dichos efectos adversos con una frecuencia tres veces mayor que quienes no fueron informados ${ }^{5}$.

Tal como ocurre con el efecto placebo, se 
estima que el efecto nocebo es frecuente y tiene una repercusión clínica perceptible que no obstante suele pasar desapercibida ${ }^{2,6}$. Pese a que consideraciones éticas hacen complejo diseñar estudios que busquen generar efectos nocebo, la aparición de éstos ha sido documentada en una serie de investigaciones y deducida a partir de los efectos negativos observados en los sujetos del grupo placebo en ensayos aleatorios doble ciego ${ }^{7}$. En cuanto a su etiología, el efecto nocebo se ha atribuido principalmente a factores psicológicos, tales como expectativas negativas del paciente $o$ a mecanismos de condicionamiento clásico, que permiten explicar porqué el efecto nocebo es más común en sujetos que antes han tenido experiencias adversas a algún tratamiento ${ }^{8}$.

Dado que la comunicación de información sobre los posibles efectos adversos de un fármaco puede desencadenar un efecto nocebo, resulta natural preguntarse por la posibilidad de omitir esta información con el fin de prevenir su potencial efecto negativo. Pero al mismo tiempo, surge el cuestionamiento ético respecto a cuánta información es posible omitir sin violar el derecho del paciente a ser bien informado y a un proceso de consentimiento informado válido. En este sentido, el concepto mismo de nocebo (del latín, "te haré daño") nos permite visualizar una forma de contradicción entre el imperativo ético de no dañar, con el deber de entregar al paciente información completa y veraz sobre su tratamiento de acuerdo con el principio de autonomía ${ }^{9}$.

\section{Respeto por la autonomía en la entrega de información}

El prudente control del médico sobre la información revelada al paciente corresponde a una práctica habitual y esperable en el contexto clínico. Resulta así comprensible que la información sobre el diagnóstico y sus alternativas de tratamiento sea comunicada de manera parcial o gradual, especialmente cuando su comprensión puede ser técnica y emotivamente difícil de asimilar. Asimismo, la manera de presentar la información tiene influencia en la percepción que el paciente tendrá de ella. Por ejemplo, cuando a pacientes se les presenta la opción entre cirugía, radioterapia o ningún tratamiento, tienden a preferir la cirugía cuando la información se les formula en términos positivos de probabilidad de sobrevida en lugar de riesgo de mortalidad $^{10}$. En este sentido, podría plantearse como conveniente ocultar información cuando se considera que su revelación puede causar daño al paciente, como ocurre con el efecto nocebo. En este caso, sin embargo, no se trata tan sólo de fraccionar o reformular la entrega de información sino de entregarla de manera incompleta. Por lo tanto, cabe preguntarse si se está cometiendo una violación del principio de autonomía del paciente al no entregarle toda la información disponible.

La omisión de información es diferente y éticamente menos cuestionable que proporcionar información engañosa. Es el caso del uso de placebo con fines terapéuticos, por ejemplo, cuando se prescriben terapias alternativas o vitaminas, señalando que tendrán un efecto beneficioso, sabiendo que no existe evidencia científica suficiente que avale estos beneficios. Aun cuando esta práctica sea bien intencionada, se incurre en un paternalismo injustificado porque el médico juzga de antemano lo que considera bueno para el paciente, en base a sus propias concepciones y base científica. La entrega de información engañosa al paciente, además de vulnerar su autonomía, puede también generar incertidumbre respecto a las prescripciones médicas, afectando de esta manera la confianza de los pacientes. Si bien en principio no es del todo rechazable el uso de placebo dada su acción terapéutica, su indicación debe ser formulada con precaución y con base empírica suficiente para presuponer su inocuidad y eficacia ${ }^{11}$.

Teniendo en cuenta estos problemas, cabe volver sobre el punto de que la entrega de información parcializada puede resultar a veces ineludible en el contexto clínico. Gran parte de la información relacionada con un diagnóstico o tratamiento puede ser irrelevante o difícil de comprender para el paciente, más aún si se realiza en un espacio limitado de tiempo. Como señalan Beauchamp y Childress, los ideales de conocimiento e independencia de un paciente plenamente autónomo son utópicos, siendo difícil determinar el límite entre lo suficiente y lo insuficiente ${ }^{12}$. Los autores sugieren "establecer cuidadosamente el límite en función de los objetivos específicos, como por ejemplo la toma de decisiones importantes [...] lo mejor es valorar si la autonomía es suficiente en cada uno de los diferentes contextos en lugar de plantear una teoría general sobre lo que es o no suficiente" (p. 116). El médico no se encuentra, por lo tanto, 
siempre en la obligación de entregar al paciente toda la información sobre su tratamiento, aunque sí debe entregar lo suficiente para que el paciente pueda eventualmente otorgar un consentimiento debidamente informado y éticamente validado según su competencia. Estas consideraciones no implican, empero, desconocer las complejidades que surgen al determinar en cada caso cuanta información es suficiente para preservar el respeto por la autonomía del paciente y minimizar al mismo tiempo los eventuales riesgos que conlleva la entrega de información.

\section{El balance entre los principios de autonomía y no-maleficencia}

Con el surgimiento de la Bioética la práctica médica se ha apartado de las tradiciones paternalistas, dando relevancia al reconocimiento de la autonomía del paciente y de su derecho a aceptar o rechazar los tratamientos médicos que se le proponen. Esto queda plasmado en la autonomía -en conjunto con los principios de beneficencia, no-maleficencia y justicia- como uno de los cuatro principios de la Bioética propuestos por Beauchamp y Childress ${ }^{12}$. De acuerdo con los autores, estos no son principios absolutos sino que guías procedimentales, prima facie moralmente aceptables y sin una jerarquización predefinida. $\mathrm{Su}$ contenido específico y la preponderancia de uno sobre otro será determinada de acuerdo al contexto y los hechos únicos de cada caso. Asimismo, al ponderar en caso de conflicto entre los principios de autonomía y no-maleficencia, se podrá recurrir a razones basadas en distintas tradiciones éticas desde las cuales se puede justificar la decisión.

Por una parte, autores de orientación liberalista como Engelhardt ${ }^{13}$ sostienen que la libertad o la autonomía del paciente tiene prioridad sobre los otros principios éticos, incluyendo aquellos que busquen maximizar la beneficencia al paciente. Esta idea se encuentra presente también en propuestas más matizadas como la de Veatch ${ }^{14}$, quien justifica el lugar preponderante de la autonomía (además de la justicia) en base a la obligación moral de respetar a las personas, por sobre consideraciones relativas a potenciales beneficios o daños. En palabras del autor, la "beneficencia y la no-maleficencia por sí mismas nunca pueden justificar incumplir una promesa, decir una mentira, violar la autonomía, matar a otro, o distribuir los bienes de manera injusta” (p. 212).

En tanto, otros enfoques recogen la tradición hipocrática de dar prioridad al beneficio del paciente. Es el caso de propuestas como la de Pellegrino y Thomasma ${ }^{15}$, quienes consideran los fines y propósitos de la actividad médica se organizan en torno al deber de ayudar, sanar y cuidar en el contexto particular de la relación médico-paciente. Para los autores, "pretensiones morales de autonomía [...] derivan de la pretensión más fundamental de preservar la integridad de la persona" (p. 131). Dado que para Pellegrino y Thomasma el nivel mínimo de preservación de esta integridad se sitúa en la no-maleficencia, podemos inferir que la prevención de dañar al paciente a través del efecto nocebo justificaría una limitación de la autonomía del paciente ${ }^{16}$.

Sin embargo interferir con la libertad del paciente para decidir sobre su tratamiento, o suplantar su voluntad con el propósito de beneficiarlo, puede ser considerado como un inapropiado paternalismo. Sin embargo, habitualmente se espera que el médico adopte una posición activa respecto a guiar o persuadir al paciente a tomar la decisión que, de acuerdo a la mejor medicina disponible, permita maximizar su bienestar y minimizar los posibles daños. Incluso, en situaciones donde se puede prever un serio daño físico, psicológico o emocional para el paciente, los códigos deontológicos en general aceptan que el médico apele al "privilegio terapéutico" de eximirse de la obligación de entregar cierta información o prescribir un tratamiento solicitado por el paciente ${ }^{17}$.

En un sentido similar, varios autores han planteado que la no-maleficencia ocupa un lugar prioritario entre los demás principios de la Bioética. Gert, Culver y Clouser ${ }^{18}$ plantean que el principio de no-maleficiencia agrupa las reglas fundacionales de la ética, las cuales giran en torno a la idea de evitar hacer el mal o causar daño. La autonomía, en cambio, se trataría de un principio relacionado con ideales morales que motivan a realizar acciones que minimicen el riesgo o la magnitud de los daños. Mientras las reglas morales relacionadas con no causar daño serían obligatorias, la autonomía corresponde a un ideal moral no exigible bajo toda circunstancia. Una propuesta similar la encontramos en la jerarquización que plantea Diego Gracia para los principios de la Bioética, donde la no-maleficencia y la justicia se 
ubican, por consenso de la comunidad, en un nivel superior de deberes definidos en la esfera pública y por lo tanto exigibles para todos sus miembros ${ }^{19}$. La autonomía, en cambio, se situaría junto con la beneficencia dentro del ámbito de deberes morales privados, propio del sistema de valores mediante el cual cada individuo busca su ideal de perfección y felicidad, y por lo tanto diverso y no exigible de manera obligatoria para todos por igual.

\section{Entrega parcial de información para prevenir efectos nocebo}

Consideramos, por tanto, que tanto el respeto por la autonomía del paciente como su derecho a un adecuado proceso de consentimiento informado se encuentran motivados por el propósito de proteger al paciente de prácticas improcedentes o potencialmente dañinas. Resulta razonable que cuando se puede prever que la revelación de información detallada puede desencadenar efectos nocebo y dañar al paciente, se realice un apropiado balance entre los principios de autonomía y no-maleficencia, sin imponer el primero sobre el segundo. Una propuesta interesante en este sentido es la de Wells y Kaptchuk relativa a la elaboración de un "consentimiento informado contextualizado" que tenga en consideración los riesgos del efecto nocebo ${ }^{5}$. El consentimiento propuesto por los autores supone considerar los posibles efectos adversos, las características personales del paciente y la enfermedad en cuestión, para adaptar la información acerca de los efectos adversos y proporcionar así la mayor transparencia minimizando a la vez de daños potenciales.

El predominio del principio de autonomía, junto a la complejidad, la sobreespecialización de la medicina y a otras características de las sociedades contemporáneas, han contribuido a que la relación médico paciente haya adquirido un carácter cuasi contractual. De esta manera, muchas veces el juicio clínico del médico se relega a un plano secundario frente al predominio de la decisión del paciente, lo que acaba constituyendo una inadecuada comprensión del principio de autonomía ${ }^{20}$. Se desconoce de esta manera la innegable asimetría entre un paciente en situación de vulnerabilidad y un profesional con la capacidad de ayudarle por medio de la curación y del cuidado. En la base de esta relación existe una alianza terapéutica, en la cual debe primar la confianza recíproca por sobre los criterios contractuales, dando lugar a decisiones compartidas en las que el paciente, según su capacidad racional y emocional, pueda aceptar o rechazar tratamientos con la ayuda y el acompañamiento del médico ${ }^{21}$. En este modelo de relación las prescripciones y la información necesitan ser graduadas y entregadas de manera prudente e individualizada para favorecer así la concordancia con los valores, expectativas y proyectos de vida del paciente.

En este marco, consideramos importante tener en consideración situaciones donde la entrega de información completa sobre eventuales efectos adversos de un tratamiento pudiera resultar inconveniente o dañina si, como efecto nocebo, genera o exacerba efectos negativos en algunos pacientes. Una manera prudente de proceder en estos casos, respetando la autonomía del paciente, puede ser la entrega de información parcializada con su consentimiento, tras explicarle los riesgos del efecto nocebo y dejando una vía de comunicación expedita para que pueda referir al médico los eventuales efectos adversos que pudiera presentar (Cf. 22). Cabe mencionar que recomendaciones similares se han planteado en el contexto de investigación biomédica, e.g. CIOMS No $10^{23}$.

Si bien es posible concluir que hay casos donde es éticamente justificable la decisión médica de entregar información parcial sobre un tratamiento, es importante señalar algunas consideraciones precautorias. Esta decisión debe estar precedida de una cuidadosa evaluación de los riesgos y beneficios del tratamiento a prescribir, considerando que el efecto nocebo puede añadir un riesgo adicional. Cabe insistir en que esta evaluación debe estar respaldada por la evidencia científica, por cuanto existe evidencia de que los médicos suelen tener expectativas imprecisas sobre los efectos adversos de sus prescripciones y tienden a subestimarlos ${ }^{24}$. Otro aspecto a enfatizar es que la decisión de ocultar información sobre efectos adversos debe ser individualizada, dado que no todos los pacientes tienen la misma predisposición a presentar respuestas nocebogénicas. Por ejemplo, se ha planteado que estas respuestas serían más frecuentes en mujeres y en sujetos con ciertas personalidades aprensivas y patologías psicosomáticas. Sin embargo, la evidencia no es concluyente respecto al perfil de los pacientes más propensos a experimentar efectos nocebo y placebo $^{25}$, por 
lo que la decisión debiera estar fundamentada en una relación de confianza con conocimiento del enfermo como persona. De cualquier manera, no resulta aceptable ocultar el riesgo de efectos adversos cuando éstos pueden tener carácter grave o irreversible, por cuanto el riesgo de presentarlos supera ampliamente el riesgo de que se generen efectos nocebo tras su comunicación (Cf. CIOMS No $10^{23}$ ).

Como para todo acto comunicativo situado en el contexto clínico, no es posible proponer un procedimiento unívoco para la entrega de información al paciente. En este sentido, las complejidades que los efectos placebo y nocebo añaden a la relación clínica nos permiten recordar que la medicina es también un arte, donde el carácter contingente de cada caso debe ser abordado de manera prudente y deliberativa.

\section{Referencias}

1. De Craen AJ, Kaptchuk TJ, Tijssen JG, Kleijnen J. Placebos and placebo effects in medicine: historical overview. Journal of the Royal Society of Medicine 1999; 92 (10): 511-5.

2. Barsky AJ, Saintfort R, Rogers MP, Borus JF. Nonspecific medication side effects and the nocebo phenomenon. Jama 2002; 287 (5): 622-7.

3. Ferreres J, Baños JE, Farré M. Efecto nocebo: la otra cara del placebo. Medicina clínica 2004; 122 (13): 511-6.

4. Hahn RA. The nocebo phenomenon: concept, evidence, and implications for public health. Preventive medicine 1997; 26 (5): 607-11.

5. Wells RE, Kaptchuk TJ. To tell the truth, the whole truth, may do patients harm: the problem of the nocebo effect for informed consent. The American Journal of Bioethics 2012; 12 (3): 22-9.

6. Petersen GL, Finnerup NB, Colloca L, Amanzio M, Price $\mathrm{DD}$, Jensen TS, et al. The magnitude of nocebo effects in pain: a meta-analysis. PAIN ${ }^{\circledR} 2014 ; 155$ (8): 1426-34.

7. Häuser W, Hansen E, Enck P. Nocebo phenomena in medicine: their relevance in everyday clinical practice. Deutsches Ärzteblatt International 2012; 109 (26): 45965.

8. Planès $S$, Villier $C$, Mallaret $M$. The nocebo effect of drugs. Pharmacology research \& perspectives 2016; 4 (2): e00208.

9. Hunter D. Could informed consent be harmful?-the problem of the nocebo effect 2012; 8 (3): 151-3.
10. Moxey A, O’Connell D, McGettigan P, Henry D. Describing treatment effects to patients. Journal of General Internal Medicine 2003; 18 (11): 948-59.

11. Alfano M. Placebo effects and informed consent. The American Journal of Bioethics 2015; 15 (10): 3-12.

12. Beauchamp TL, Childress J. Principios de Bioética. Ed. Mason SA. Barcelona. España. 1999.

13. Engelhardt HT. The foundations of bioethics. Oxford University Press; 1996.

14. Veatch RM. Resolving conflicts among principles: ranking, balancing, and specifying. Kennedy Institute of Ethics Journal 1995; 5 (3): 199-218.

15. Pellegrino ED, Thomasma DC. The virtues in medical practice. Oxford University Press on Demand; 1993.

16. Pellegrino ED, Thomasma DC. The conflict between autonomy and beneficence in medical ethics: proposal for a resolution. J Contemp Health Law Policy 1987; 3: 23.

17. Salinas R, Echeverría C, Arriagada A, Goic A, Quintana C, Rojas A, et al . Does therapeutic privilege have a place in modern medicine? Rev Med Chile 2017; 145 (9): 1198-202.

18. Gert B, Culver CM, Clouser KD, Bioethics A. A return to fundamentals. Oxford. Oxford University Press. 1997.

19. Gracia D. Fundamentación y enseñanza de la bioética. Bogotá: Editorial El Búho; 1998.

20. Stirrat GM, Gill R. Autonomy in medical ethics after O’Neill. Journal of Medical Ethics 2005; 31 (3): 127-30.

21. Rodríguez A. Modelos de la relación médico-paciente reflejo de la deshumanización de la salud. ARS MEDICA Revista de Ciencias Médicas 2006; 35 (1): 55-62.

22. Miller FG, Colloca L. The placebo phenomenon and medical ethics: Rethinking the relationship between informed consent and risk-benefit assessment. Theoretical medicine and bioethics 2011; 32 (4): 229-43.

23. Organización Panamericana de la Salud y Consejo de Organizaciones Internacionales de las Ciencias Médica. Pautas éticas internacionales para la investigación relacionada con la salud con seres humanos, Cuarta Edición. Ginebra: Consejo de Organizaciones Internacionales de las Ciencias Médicas (CIOMS); 2016.

24. Hoffmann TC, Del Mar C. Clinicians' expectations of the benefits and harms of treatments, screening, and tests: a systematic review. JAMA internal medicine 2017; 177 (3): 407-19.

25. Schedlowski M, Enck P, Rief W, Bingel U. Neuro-bio-behavioral mechanisms of placebo and nocebo responses: implications for clinical trials and clinical practice. Pharmacological reviews 2015; 67 (3): 697730 . 